\title{
Plasmon slot waveguides: Towards chip-scale propagation with subwavelength-scale localization
}

\author{
J. A. Dionne,* L. A. Sweatlock, and H. A. Atwater \\ Thomas J. Watson Laboratories of Applied Physics, California Institute of Technology, MC 128-95, Pasadena, California 91125, USA \\ A. Polman \\ Thomas J. Watson Laboratories of Applied Physics, California Institute of Technology, MC 128-95, Pasadena, California 91125 , USA \\ and The Center for Nanophotonics, FOM-Institute AMOLF, Kruislaan 407, 1098 SJ Amsterdam, The Netherlands
}

(Received 9 July 2005; revised manuscript received 2 November 2005; published 5 January 2006)

\begin{abstract}
We present a numerical analysis of surface plasmon waveguides exhibiting both long-range propagation and spatial confinement of light with lateral dimensions of less than $10 \%$ of the free-space wavelength. Attention is given to characterizing the dispersion relations, wavelength-dependent propagation, and energy density decay in two-dimensional $\mathrm{Ag} / \mathrm{SiO}_{2} / \mathrm{Ag}$ structures with waveguide thicknesses ranging from $12 \mathrm{~nm}$ to $250 \mathrm{~nm}$. As in conventional planar insulator-metal-insulator (IMI) surface plasmon waveguides, analytic dispersion results indicate a splitting of plasmon modes-corresponding to symmetric and antisymmetric electric field distributions- as $\mathrm{SiO}_{2}$ core thickness is decreased below $100 \mathrm{~nm}$. However, unlike IMI structures, surface plasmon momentum of the symmetric mode does not always exceed photon momentum, with thicker films $(d \sim 50 \mathrm{~nm})$ achieving effective indices as low as $n=0.15$. In addition, antisymmetric mode dispersion exhibits a cutoff for films thinner than $d=20 \mathrm{~nm}$, terminating at least $0.25 \mathrm{eV}$ below resonance. From visible to near infrared wavelengths, plasmon propagation exceeds tens of microns with fields confined to within $20 \mathrm{~nm}$ of the structure. As the $\mathrm{SiO}_{2}$ core thickness is increased, propagation distances also increase with localization remaining constant. Conventional waveguiding modes of the structure are not observed until the core thickness approaches $100 \mathrm{~nm}$. At such thicknesses, both transverse magnetic and transverse electric modes can be observed. Interestingly, for nonpropagating modes (i.e., modes where propagation does not exceed the micron scale), considerable field enhancement in the waveguide core is observed, rivaling the intensities reported in resonantly excited metallic nanoparticle waveguides.
\end{abstract}

DOI: 10.1103/PhysRevB.73.035407

PACS number(s): 73.20.Mf

\section{INTRODUCTION}

Photonics has experienced marked development with the emergence of nanoscale fabrication and characterization techniques. This progress has brought with it a renewed interest in surface plasmons (SPs) - electron oscillations that allow electromagnetic energy to be localized, confined, and guided on subwavelength scales. Waveguiding over distances of $0.5 \mu \mathrm{m}$ has been demonstrated in linear chains of metal nanoparticles, ${ }^{1}$ and numerous theoretical and experimental studies $^{2-5}$ indicate the possibility of multicentimeter plasmon propagation in thin metallic films. Moreover, the locally enhanced field intensities observed in plasmonic structures promise potential for molecular biosensing, ${ }^{5-10}$ surface enhanced Raman spectroscopy, ${ }^{11-13}$ and nonlinear optical device applications. ${ }^{14-18}$

In planar metallodielectric geometries, surface plasmons represent plane-wave solutions to Maxwell's equations, with the complex wave vector determining both field symmetry and damping. For bound modes, field amplitudes decay exponentially away from the metal/dielectric interface with field maxima occurring at the surface. ${ }^{19}$ While the dispersion properties of long-ranging SPs mimic those of a photon, multicentimeter propagation is often accompanied by significant field penetration into the surrounding dielectric. For thin Ag films $(\sim 10 \mathrm{~nm})$ excited at telecommunications frequencies, electric field skin depths can exceed $5 \mu \mathrm{m} .{ }^{3,4}$ In terms of designing highly integrated photonic and plasmonic struc- tures, a more favorable balance between localization and loss is required.

While metals are characteristically lossy, the bound SP modes of a single metal/dielectric interface can propagate over several microns under optical illumination. ${ }^{4,19}$ In such a geometry, the field skin depth increases exponentially with wavelength in the dielectric but remains approximately constant $(\sim 25 \mathrm{~nm})$ in the metal for visible and near-infrared excitation frequencies. This observation has inspired a new class of plasmon waveguides that consist of an insulating core and conducting cladding. Not unlike conventional waveguides (including dielectric slab waveguides at optical frequencies, metallic slot waveguides at microwave frequencies, and the recently proposed semiconductor slot waveguides of Ref. 20), these metal-insulator-metal (MIM) structures guide light via the refractive index differential between the core and cladding. However, unlike dielectric slot waveguides, both plasmonic and conventional waveguiding modes can be accessed, depending on transverse core dimensions. MIM waveguides may thus allow optical mode volumes to be reduced to subwavelength scales - with minimal field decay out of the waveguide physical cross sectioneven for frequencies far from the plasmon resonance.

Several theoretical studies have already investigated surface plasmon propagation and confinement in MIM structures. $^{21,22}$ However, few studies have investigated wavelength-dependent MIM properties arising from realistic models for the complex dielectric function of metals. The critical dependence of waveguiding experiments on excita- 
tion wavelength and surface plasmon frequency renders such an analysis essential. In this paper, we discuss the surface plasmon and conventional waveguiding modes of MIM structures, characterizing the metal by the empirical optical constants of Johnson and Christy ${ }^{23}$ and numerically determining the dispersion, propagation, and localization for both field symmetric and antisymmetric modes.

\section{PLASMON SLOT WAVEGUIDE DISPERSION}

The eigenmodes of planar multilayer structures may be solved via the vector wave equation under constraint of tangential $\mathbf{E}$ and normal $\mathbf{D}$ field continuity. Uniqueness of the results is guaranteed by the Helmholtz theorem. For unpolarized waves in a three-layer symmetric structure, the electromagnetic fields take the form

$$
\begin{aligned}
& E(x, z, t)=\left(E_{x} \hat{x}+E_{y} \hat{y}+E_{z} \hat{z}\right) e^{i\left(k_{x} x-\omega t\right)} \\
& B(x, z, t)=\left(B_{x} \hat{x}+B_{y} \hat{y}+B_{z} \hat{z}\right) e^{i\left(k_{x} x-\omega t\right)},
\end{aligned}
$$

with $E_{y}, B_{x}$, and $B_{z}$ identically zero for transverse magnetic (TM) polarization and $E_{x}, E_{z}$, and $B_{y}$ identically zero for transverse electric (TE) polarization.

Inside the waveguide, the field components may be written as:

$$
\begin{gathered}
E_{x}^{i n}=e^{-i k_{z 1} z} \pm e^{i k_{z 1} z}, \\
E_{y}^{i n}=0, \\
E_{z}^{i n}=\left(\frac{k_{x}}{k_{z 1}}\right)\left(e^{-i k_{z 1} z} \mp e^{i k_{z 1} z}\right), \\
B_{x}^{i n}=0, \\
B_{y}^{i n}=\left(\frac{-\omega \varepsilon_{1}}{c k_{x}}\right)\left(e^{-i k_{z 1} z} \mp e^{i k_{z 1} z}\right), \\
B_{z}^{i n}=0,
\end{gathered}
$$

for the TM polarization and as:

$$
\begin{gathered}
E_{x}^{i n}=0, \\
E_{y}^{i n}=e^{i k_{z 1} z} \pm e^{-i k_{z 1} z}, \\
E_{z}^{i n}=0, \\
B_{x}^{i n}=\left(\frac{-k_{z 1} c}{\omega}\right)\left(e^{i k_{z 1} z} \mp e^{-i k_{z 1} z}\right), \\
B_{y}^{i n}=0, \\
B_{z}^{i n}=\left(\frac{k_{x} c}{\omega}\right)\left(e^{i k_{z 1} z} \pm e^{-i k_{z 1} z}\right) .
\end{gathered}
$$

for the TE polarization. Outside the guide, the components are given by:

$$
\begin{gathered}
E_{x}^{\text {out }}=\left(e^{-i k_{z 1} d / 2} \pm e^{i k_{z 1} d / 2}\right) e^{i k_{z 2}(z-d / 2)}, \\
E_{y}^{\text {out }}=0, \\
E_{z}^{\text {out }}=\left(\frac{\varepsilon_{1} k_{x}}{\varepsilon_{2} k_{z 1}}\right)\left(e^{-i k_{z 1} d / 2} \mp e^{i k_{z 1} d / 2}\right) e^{i k_{z 2}(z-d / 2)}, \\
B_{x}^{\text {in }}=0, \\
B_{y}^{\text {out }}=\left(\frac{-\omega \varepsilon_{1}}{c k_{x}}\right)\left(e^{-i k_{z 1} d / 2} \mp e^{i k_{z 1} d / 2}\right) e^{i k_{z 2}(z-d / 2)}, \\
B_{z}^{\text {out }}=0,
\end{gathered}
$$

for the TM polarization and as:

$$
\begin{gathered}
E_{x}^{\text {out }}=0, \\
E_{y}^{\text {out }}=\left(e^{i k_{z 1} d / 2} \pm e^{-i k_{z 1} d / 2}\right) e^{i k_{z 2}(z-d / 2)}, \\
E_{z}^{\text {out }}=0, \\
B_{x}^{\text {out }}=\left(\frac{-k_{z 1} c}{\omega}\right)\left(e^{i k_{z 1} d / 2} \mp e^{-i k_{z 1} d / 2}\right) e^{i k_{z 2}(z-d / 2),} \\
B_{y}^{\text {out }}=0, \\
B_{z}^{\text {out }}=\left(\frac{k_{x} c}{\omega}\right)\left(e^{i k_{z 1} d / 2} \pm e^{-i k_{z 1} d / 2}\right) e^{i k_{z 2}(z-d / 2)} .
\end{gathered}
$$

for the TE polarization. The in-plane wave vector $k_{x}$ is defined by the dispersion relations:

$$
\begin{aligned}
& L+:\left\{\begin{array}{c}
\varepsilon_{1} k_{z 2}+\varepsilon_{2} k_{z 1} \tanh \left(\frac{-i k_{z 1} d}{2}\right)=0 T M \\
k_{z 2}+k_{z 1} \tanh \left(\frac{-i k_{z 1} d}{2}\right)=0 T E
\end{array}\right. \\
& L-:\left\{\begin{array}{c}
\varepsilon_{1} k_{z 2}+\varepsilon_{2} k_{z 1} \operatorname{coth}\left(\frac{-i k_{z 1} d}{2}\right)=0 T M \\
k_{z 2}+k_{z 1} \operatorname{coth}\left(\frac{-i k_{z 1} d}{2}\right)=0 T E
\end{array}\right.
\end{aligned}
$$

with $k_{z}$ defined by momentum conservation:

$$
k_{z 1,2}^{2}=\varepsilon_{1,2}\left(\frac{\omega}{c}\right)^{2}-k_{x}^{2} .
$$

The analysis assumes the structure is centered at $z=0$ with core thickness $d$ and wave propagation occurring along the positive $x$ direction (see Fig. 1). The core (cladding) is composed of material with complex dielectric constant $\varepsilon_{1}\left(\varepsilon_{2}\right)$; we assume all materials are nonmagnetic so that the magnetic permeability $\mu$ has been taken equal to 1 . Since surface plasmons represent charge density oscillations, the dispersion relations of Eqs. (6) and (7) define tangential electric field configurations that are either antisymmetric $(L+)$ or 


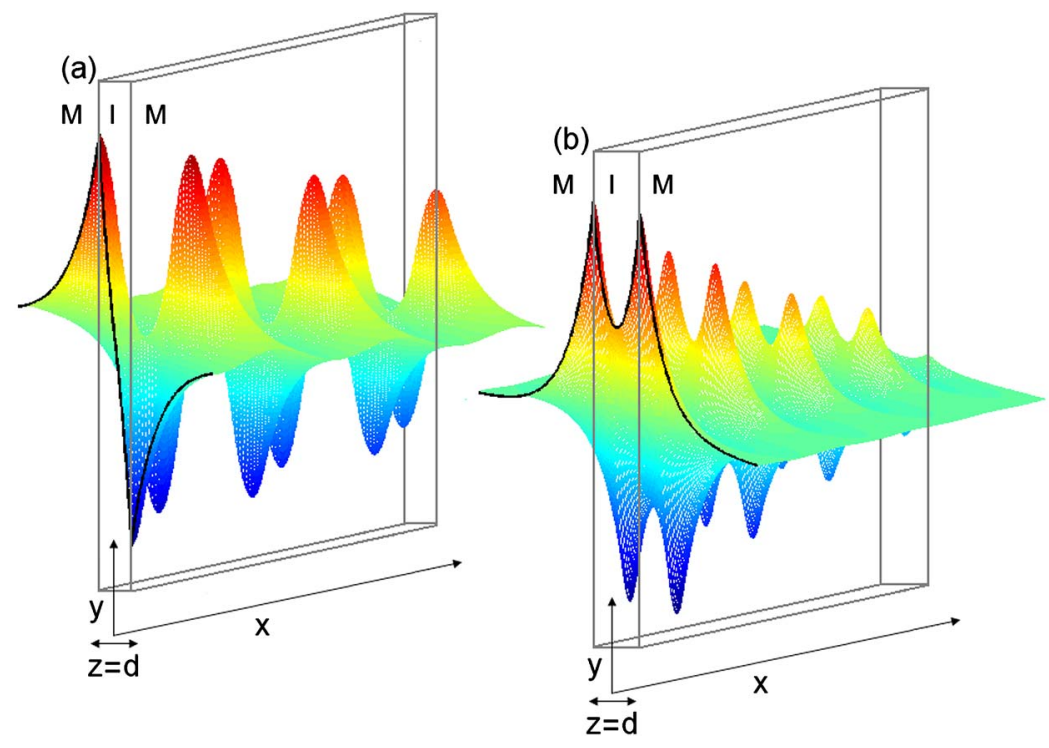

FIG. 1. (Color online) Geometry and characteristic tangential $(x)$ electric field profiles for MIM slot waveguides. The waveguide is centered at $z=0$ with core thickness $d$ and wave propagation occurring along the positive $x$ direction. (a) Field antisymmetric mode, (b) Field symmetric mode.

symmetric $(L-)$ with respect to the waveguide median. TE surface plasmon waves do not generally exist in planar metallodielectric structures, since continuity of $E_{y}$ forbids charge accumulation at the interface.

When a plasma is excited at a metallodielectric interface, electrons in the metal create a surface polarization that gives rise to a localized electric field. In insulator-metal-insulator (IMI) structures, electrons of the metallic core screen the charge configuration at each interface and maintain a nearzero (or minimal) field within the waveguide. As a result, the surface polarizations on either side of the metal film remain in phase and a cutoff frequency is not observed for any transverse waveguide dimension. In contrast, screening does not occur within the dielectric core of MIM waveguides. At each metal-dielectric interface, surface polarizations arise and evolve independently of the other interface, and plasma oscillations need not be energy- or wave-vector-matched to each other. Therefore, for certain MIM dielectric core thicknesses, interface SPs may not remain in phase but will exhibit a beating frequency; as transverse core dimensions are increased, "bands" of allowed energies or wave vectors and "gaps" of forbidden energies will be observed.

This behavior is illustrated in Fig. 2, which plots the TM dispersion relations for an MIM waveguide with core thicknesses of $250 \mathrm{~nm}$ [Fig. 2(a)] and $100 \mathrm{~nm}$ [Fig. 2(b)]. Since TE modes in MIM guides resemble those of a conventional dielectric core, conducting cladding waveguide, their dispersion is not explicitly considered here. The waveguide consists of a three-layer metallodielectric stack with a $\mathrm{SiO}_{2}$ core and a Ag cladding. The metal is defined by the empirical optical constants of Johnson and Christy ${ }^{23}$ and the dielectric constant for the oxide is adopted from Palik's handbook. ${ }^{24}$ Solution of the dispersion relations [Eqs. (6) and (7)] was achieved via application of a Nelder-Mead minimization routine in complex wave-vector space; details of implementation and convergence properties are described elsewhere. ${ }^{4}$ For reference, the figures include the waveguide TM dispersion curve in the limit of infinite core thickness, plotted in black. Allowed wave vectors are seen to exist for all freespace wavelengths (energies) and exhibit exact agreement with the dispersion relation for a single $\mathrm{Ag} / \mathrm{SiO}_{2}$ interface SP.

Figure 2(a) plots the "bound" modes (here, modes occurring at frequencies below the SP resonance, see Ref. 25) of a $\mathrm{Ag} / \mathrm{SiO}_{2} / \mathrm{Ag}$ waveguide with core thickness $d=250 \mathrm{~nm}$. The asymmetric bound $\left(a_{b}\right)$ modes correspond to solution of $L+$ and are plotted in light gray; the symmetric bound $\left(s_{b}\right)$ modes correspond to solution of $L-$ and are plotted in dark gray. As seen, multiple bands of allowed and forbidden frequencies are observed. The allowed $a_{b}$ modes follow the light line for energies below $\sim 1 \mathrm{eV}$ and resemble conventional dielectric core, conducting cladding waveguide modes for energies above $\sim 2.8 \mathrm{eV}$. Tangential electric fields $E_{x}$ in each $a_{b}$ regime are plotted in the inset and highlight the distinction in mode localization: At $410 \mathrm{~nm}$, the mode is localized within the waveguide core and resembles a conventional TM waveguide profile. At $1.7 \mu \mathrm{m}$, dispersion is more plasmonlike and field maxima of the mode occur at each metal-dielectric interface.

In contrast to the $a_{b}$ modes, the $s_{b}$ modes are only observed for energies between 1.5 and $3.2 \mathrm{eV}$. Dispersion for this mode is reminiscent of conventional dielectric core, dielectric cladding waveguides, with end-point asymptotes corresponding to tangent line slopes (effective indices) of $n$ $=8.33$ at $1.5 \mathrm{eV}$ and $n=4.29$ at $3.2 \mathrm{eV}$. For energies exceeding $\sim 2.8 \mathrm{eV}$, wave vectors of the $s_{b}$ mode are matched with those of the SP and the tangential electric field transits from a core mode to an interface mode [see the $1 \mathrm{st}(\sim 3 \mathrm{eV})$ and 3rd $(\sim 1.9 \mathrm{eV})$ panels of the inset]. As the core layer thickness is increased through $1 \mu \mathrm{m}$ (data not shown), the number of $a_{b}$ and $s_{b}$ bands increases with the $a_{b}$ modes generally lying at higher energies. In analogy with conventional waveguides, larger (but bounded) core dimensions increase the number of modes supported by the structure.

Figure 2(b) plots the bound mode dispersion curves for an MIM waveguide with $\mathrm{SiO}_{2}$ core thickness $d=100 \mathrm{~nm}$. Again, the allowed $a_{b}$ modes are plotted in light gray while the allowed $s_{b}$ modes are dark gray. Although the $s_{b}$ mode resembles conventional waveguide dispersion, the $a_{b}$ mode is seen to exhibit plasmonlike behavior. Accordingly, the con- 

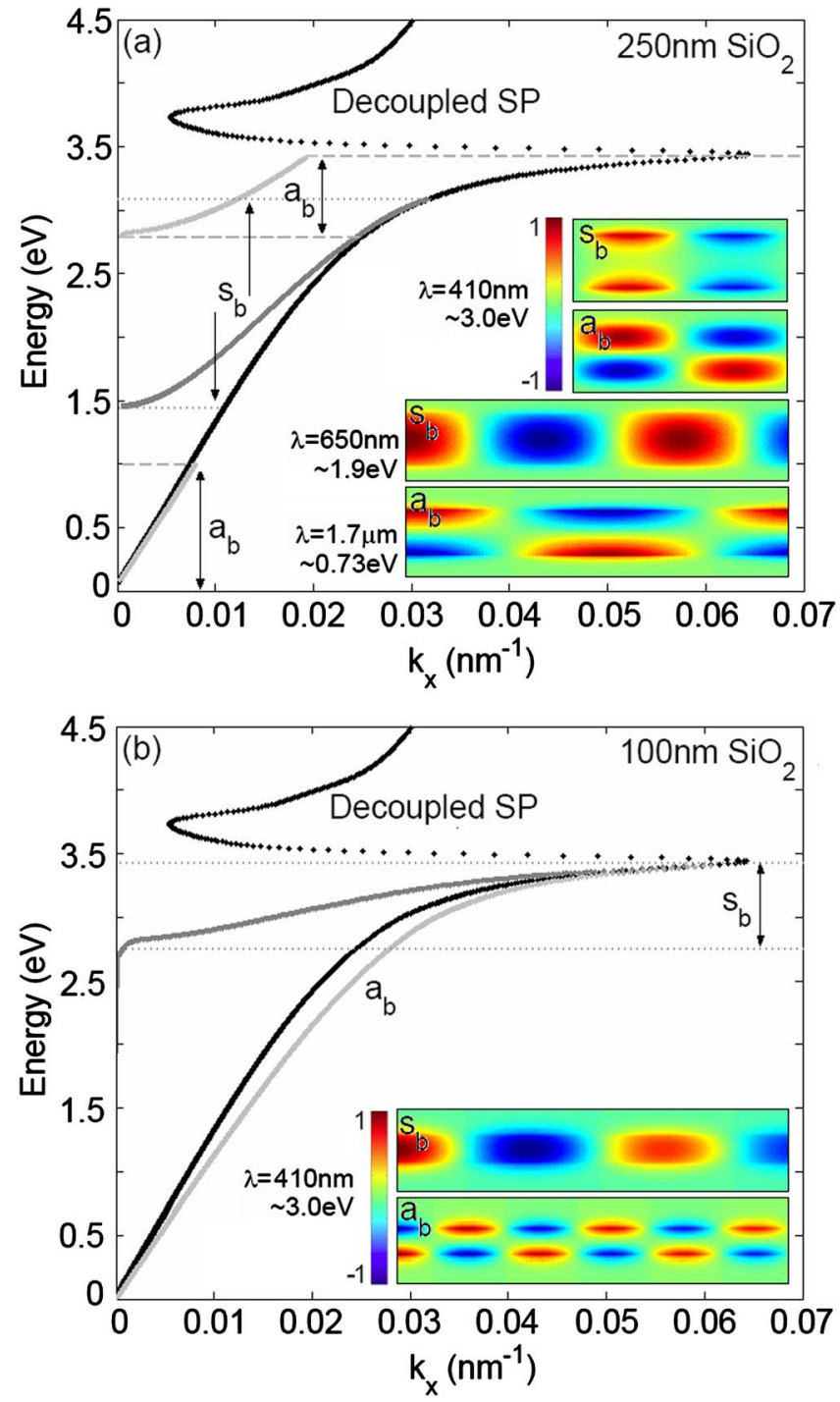

FIG. 2. (Color online) Transverse magnetic dispersion relations and tangential electric field $\left(E_{x}\right)$ profiles for MIM planar waveguides with a $\mathrm{SiO}_{2}$ core and a $\mathrm{Ag}$ cladding. Dispersion of an infinitely thick core is plotted in black and is in exact agreement with results for a single $\mathrm{Ag} / \mathrm{SiO}_{2}$ interface plasmon. (a) For oxide thicknesses of $250 \mathrm{~nm}$, the structure supports conventional waveguiding modes with cutoff wave vectors observed for both the symmetric ( $s_{b}$, dark gray) and antisymmetric ( $a_{b}$, light gray) field configurations. (b) As oxide thickness is reduced to $100 \mathrm{~nm}$, both conventional and plasmon waveguiding modes are supported. Accordingly, tangential electric fields $\left(E_{x}\right)$ are localized within the core for conventional modes but propagate along the metal-dielectric interface for plasmon modes [inset, plotted in (a) at free-space wavelengths of $\lambda=410 \mathrm{~nm}(\sim 3 \mathrm{eV})$ (top two panels), $\lambda=650 \mathrm{~nm}$ $(\sim 1.9 \mathrm{eV})$, and $\lambda=1.7 \mu \mathrm{m}(\sim 0.73 \mathrm{eV})$, and in (b) at $\lambda=410 \mathrm{~nm}$ $(\sim 3 \mathrm{eV})]$.

ventional waveguiding modes are found only at higher energies (over a range of $\sim 1 \mathrm{eV}$ ), where photon wavelengths are small enough to be guided by the structure. The inset shows snapshots of the tangential electric field for both modes at a free-space wavelength $\lambda=410 \mathrm{~nm}(\sim 3 \mathrm{eV})$. As seen, the $s_{b}$ field is concentrated in the waveguide core with minimal penetration into the conducting cladding. In contrast, the $a_{b}$ field is highly localized at the surface, with field penetration approximately symmetric on each side of the metal-dielectric interface. The presence of both conventional and SP waveguiding modes represents a transition to subwavelength-scale photonics. Provided momentum can be matched between the photon and the SP, and energy will be guided in a polariton mode along the metal-dielectric interface. Otherwise, the structure will support a conventional waveguide mode, but propagation will only occur over a narrow frequency band.

As MIM core thickness is reduced below $100 \mathrm{~nm}$, the structure can no longer serve as a conventional waveguide. Light impinging the structure will diffract and decay evanescently, unless it is coupled into a SP mode. Figure 3 illustrates the TM dispersion of such subwavelength structures as core thickness is varied from $50 \mathrm{~nm}$ down to $12 \mathrm{~nm}$. As in Fig. 2, the dispersion curve in the limit of infinite core thickness is also included (black curve).

Figure 3(a) plots the TM dispersion relation for the symmetric electric-field bound modes with $d=50,35,20$, and $12 \mathrm{~nm}$. Insets plot the tangential electric field profiles for $d$ $=50 \mathrm{~nm}$ and $d=12 \mathrm{~nm}$ at free-space wavelengths of $\lambda$ $=1.55 \mu \mathrm{m}(\sim 0.8 \mathrm{eV})$ and $\lambda=350 \mathrm{~nm}(\sim 3.5 \mathrm{eV})$, respectively. Functionally, the dispersion behaves like the thin-film $s_{b}$ modes of the IMI guide, with larger wave vectors achieved at lower energies for thinner films. As free-space energies approach SP resonance, the wave vector reaches its maximum value before cycling through the higher energy "quasibound" modes (not shown; see Ref. 4). A 12-nm-thick oxide can reach wave vectors as high as $k_{x}=0.2 \mathrm{~nm}^{-1}\left(\lambda_{\mathrm{sp}}\right.$ $=31 \mathrm{~nm}$ ), comparable to the resonant wave vectors observed in $12 \mathrm{~nm}$ Ag IMI waveguides. However, unlike IMI structures, the dispersion curve does not lie entirely below the single-interface (thick-film) limit. Over a finite energy bandwidth, SP momentum exceeds photon momentum both in $\mathrm{SiO}_{2}$ and in vacuum. The 50 -nm-thick oxide provides the most striking example of this behavior: dispersion lies completely to the left of the decoupled SP mode. In addition, the low-energy asymptotic behavior follows a light line corresponding to a refractive index of $n=0.15$. This low effective index suggests that polariton modes of MIM structures more highly sample the imaginary component of the metal dielectric function than the core dielectric function. In the lowenergy limit, the $s_{b}$ SP truly represents a photon trapped on the metal surface.

Figure 3(b) plots the TM dispersion relation for the antisymmetric electric-field bound modes, again with $d=50,35$, 20 , and $12 \mathrm{~nm}$. The tangential electric field profile (see inset, plotted for $d=50 \mathrm{~nm}$ at $\lambda=1.55 \mu \mathrm{m}$ ) confirms the purely plasmonic nature of the mode. In contrast with IMI structures, where $a_{b}$ dispersion approaches the light line for thinner films, wave vectors of the MIM structure achieve larger values at lower energies. For energies well below SP resonance $\left(\omega_{\mathrm{sp}}\right)$, the observed behavior is not unlike the $s_{b}$ modes of IMI guides. However, at higher energies, bound mode dispersion does not always terminate at $\omega_{\text {sp. }}$. In fact, cutoff frequencies occur at least $0.25 \mathrm{eV}$ below SP resonance for core thicknesses of less than $20 \mathrm{~nm}$. And, while the maximum wave vector always exceeds the decoupled SP resonance wave vector, the cutoff $k_{x}$ for $d \leqslant 20 \mathrm{~nm}$ remains es- 

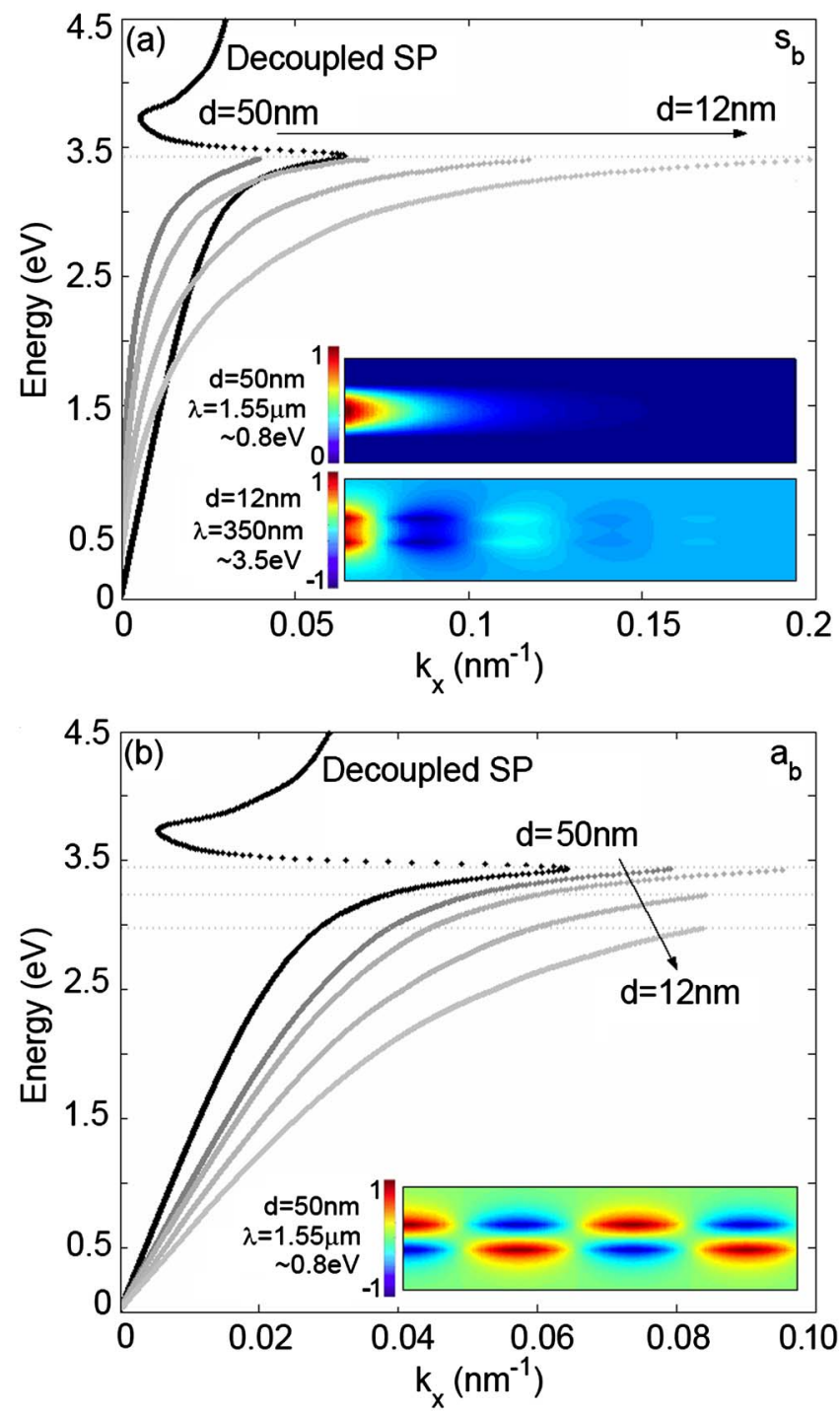

FIG. 3. (Color online) Transverse magnetic dispersion relations and tangential electric field $\left(E_{x}\right)$ snapshots of MIM $\left(\mathrm{Ag} / \mathrm{SiO}_{2} / \mathrm{Ag}\right)$ structures as oxide thickness $d$ is varied between $12 \mathrm{~nm}, 20 \mathrm{~nm}$, $35 \mathrm{~nm}$, and $50 \mathrm{~nm}$. As in Fig. 1, dispersion for an infinitely thick oxide core is plotted in black. While both the field symmetric (a) and antisymmetric (b) modes exhibit plasmonlike dispersion, the results do not parallel the behavior observed in IMI waveguides. In (a), the symmetric mode does not lie entirely below the thick-film limit, and SP momentum can exceed photon momentum both in $\mathrm{SiO}_{2}$ and in vacuum. In (b), the antisymmetric mode is seen to exhibit dispersion similar to the symmetric mode of IMI guides. However, as $d$ decreases below $20 \mathrm{~nm}$, dispersion no longer terminates on resonance, a result of skin-depth effects related to the Goos-Hanchen shift.

sentially unchanged. An explanation is provided by the Goos-Hanchen effect. As a signal propagates along the guide, it undergoes total internal reflection with slight phase shifts induced by field penetration into and out of the metal cladding. For thinner films, waveguide dimensions are comparable to the field skin depth $(\sigma)$ in the metal. Moreover, the $a_{b} E_{x}$ field distribution exhibits a node at the waveguide median so that energy densities are more highly concentrated at

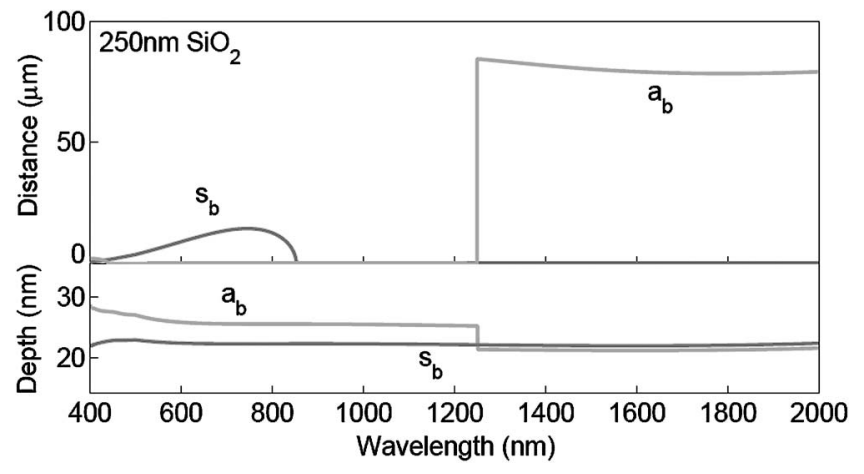

FIG. 4. $\mathrm{MIM}\left(\mathrm{Ag} / \mathrm{SiO}_{2} / \mathrm{Ag}\right) \mathrm{TM}$-polarized propagation and skin depth plotted as a function of wavelength for a core thickness of $d=250 \mathrm{~nm}$. Both $s_{b}$ and $a_{b}$ modes are observed and exhibit cutoff in accordance with the dispersion curve of Fig. 2. Propagation lengths of conventional (as opposed to plasmonic) waveguiding modes are recovered and correlated with skin depth.

the metal surface. As waveguide dimensions are decreased, this enhanced field in the metal magnifies Goos-Hanchen contributions significantly. In the limit of $d \leqslant \sigma$, complete SP dephasing could result.

\section{MODE PROPAGATION AND SKIN DEPTH}

Surface plasmon dispersion and propagation are governed by the real and imaginary components, respectively, of the in-plane wave vector. Generally, propagation is high in regimes of near-linear dispersion where high signal velocities overcome internal loss mechanisms. In IMI structures, multicentimeter propagation is observed for near-infrared wavelengths where dispersion follows the light line. However, this long-range propagation is achieved at the expense of confinement: transverse field penetration typically exceeds microns in the surrounding dielectric. In MIM structures, SP penetration into the cladding will be limited by the skin depth of optical fields in the metal. This restriction motivates the question of how skin depth affects propagation, particularly for thin films.

\section{Propagation for the transverse magnetic polarization}

Figures 4 and 5 illustrate the interdependence of skin depth and propagation in MIM structures for film thicknesses from $12-250 \mathrm{~nm}$. The top panels plot propagation of the TM modes for the structure as a function of free-space wavelength; the bottom panels plot the corresponding skin depth. Figure 4 plots propagation and skin depth for a $250 \mathrm{~nm}$ oxide layer. In accordance with the dispersion relations, wave propagation exhibits allowed and forbidden bands for the symmetric and antisymmetric modes. The $s_{b}$ mode is seen to propagate for wavelengths between 400 and $850 \mathrm{~nm}$, with maximum propagation distances of $\sim 15 \mu \mathrm{m}$. The skin depth for this mode is approximately constant over all wavelengths, never exceeding $22 \mathrm{~nm}$ in the metal. ${ }^{26}$ In contrast, the $a_{b}$ mode is seen to propagate distances of $80 \mu \mathrm{m}$ for wavelengths greater than $1250 \mathrm{~nm}$. For wavelengths below $450 \mathrm{~nm}$, a smaller band of propagation is also observed, 

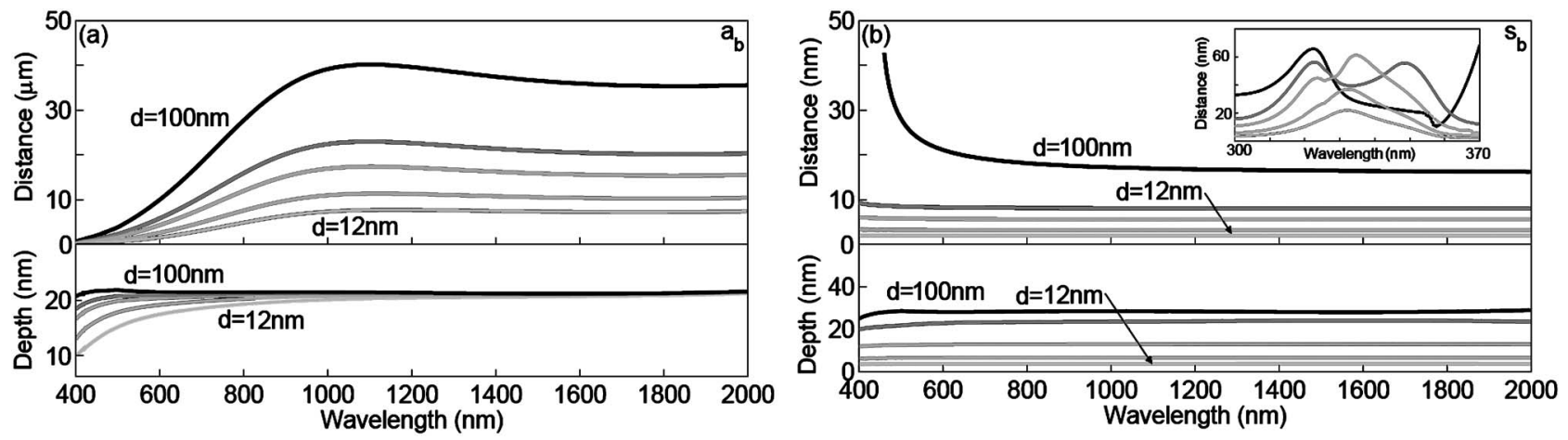

FIG. 5. MIM (Ag/ $\left./ \mathrm{SiO}_{2} / \mathrm{Ag}\right)$ TM-polarized propagation and skin depth plotted as a function of wavelength for core thicknesses of $d$ $=12 \mathrm{~nm}, 20 \mathrm{~nm}, 35 \mathrm{~nm}, 50 \mathrm{~nm}$, and $100 \mathrm{~nm}$. In panel (a), the field antisymmetric modes of MIM guides are seen to propagate over $10 \mu \mathrm{m}$ with skin depth never exceeding approximately $20 \mathrm{~nm}$. In (b), the symmetric modes of thinner films $(d \leqslant 50 \mathrm{~nm})$ remain evanescent for all wavelengths. However, as $d$ approaches $100 \mathrm{~nm}$, conventional waveguiding modes can be accessed, and a region of enhanced propagation is observed for $\lambda \leqslant 400 \mathrm{~nm}$. Inset: Propagation distances of the symmetric mode for wavelengths characteristic of the quasi-bound regime. The dissociation of the thin-film single peak to the thick-film double peak indicates the onset of conventional waveguiding.

though distances do not exceed $2 \mu \mathrm{m}$. In regions of high propagation (i.e., above $1250 \mathrm{~nm}$ ), skin depth remains approximately constant at $20 \mathrm{~nm}$; below $1250 \mathrm{~nm}$, however, skin depths approach $30 \mathrm{~nm}$. Interestingly, the figure indicates only a slight correlation between propagation and skin depth for both the $a_{b}$ and $s_{b}$ modes. This relation suggests that the metal (i.e., absorption) is not the limiting loss mechanism for wave propagation in MIM structures. ${ }^{27}$

Figure 5(a) illustrates the propagation distance and skin depth for the antisymmetric bound mode for oxide thicknesses from $12-100 \mathrm{~nm}$. The continuous plasmonlike dispersion relations of Figs. 2(b) and 3(b) are well correlated with the observed propagation: decay lengths are longest for larger wavelengths, where dispersion follows the light line. Plasmon propagation generally increases with increasing film thickness, approaching $\sim 10 \mu \mathrm{m}$ for a 12 -nm oxide layer and $\sim 40 \mu \mathrm{m}$ for a 100 -nm-thick oxide. Nevertheless, field penetration remains approximately constant in the $\mathrm{Ag}$ cladding, never exceeding $20 \mathrm{~nm}$. Thus, unlike IMI plasmon waveguides, MIM waveguides can achieve micron-scale propagation with nanometer-scale confinement.

Figure 5(b) plots propagation and skin depth for the symmetric bound modes of thin films. As with the $a_{b}$ modes, larger oxide thicknesses support increased propagation distances. However, the wave remains evanescent for thicknesses up through $50 \mathrm{~nm}$, with propagation not exceeding $10 \mathrm{~nm}$ for longer wavelengths. $\mathrm{As}_{\mathrm{SiO}_{2}}$ thicknesses approach $100 \mathrm{~nm}$, a band of allowed propagation is observed at higher frequencies, reflecting the dispersion of Fig. 2(b): at $\lambda$ $=400 \mathrm{~nm}$, propagation lengths are as high as $0.5 \mu \mathrm{m}$. In addition, thin films exhibit a local maximum in propagation for wavelengths corresponding to the transition between quasibound and radiative modes (see inset), analogous to IMI guides. ${ }^{4}$ For films with $d<35 \mathrm{~nm}$, only a single peak is observed. However, as film thickness is increased, the peak begins to split, with the lower energy peak forming the first band of allowed propagation. The transition indicates a dissociation of the quasibound modes and marks the onset of conventional waveguiding. While this regime is characterized by a slight increase in skin depth, field penetration for a given $d$ remains generally constant over the full wavelength range. Thus, unlike IMI structures, extinction is determined not by ohmic losses but by field interference upon phase shifts induced by the metal. Whether MIM structures support propagating modes or purely evanescent fields, skin depth is limited by absorption and will not exceed $30 \mathrm{~nm}$.

\section{Existence of transverse electric MIM modes}

Surface plasmons are generally transverse magnetic in nature, with interface charges allowed by the discontinuity of $E_{z}$. In planar IMI waveguides, transverse electric SP modes are not supported since $E_{y}$ is continuous. However, the existence of conventional and SP modes in MIM guides suggests that TE waves might propagate for certain oxide core thicknesses and excitation wavelengths. Figure 6 illustrates TE propagation for MIM waveguides with core thicknesses of $12 \mathrm{~nm}$ through $250 \mathrm{~nm}$. Panels 6(a) and 6(b) plot propagation of the $a_{b}$ mode, with the top panel depicting thicker oxide propagation $(d=100 \mathrm{~nm}, 150 \mathrm{~nm}, 175 \mathrm{~nm}, 250 \mathrm{~nm})$ and the bottom panel depicting thin film propagation $(d$ $=12-50 \mathrm{~nm}$ ). Panels 6(b) and 6(c) plot propagation of the $s_{b}$ mode.

As seen in panels 6(a) and 6(c), thicker oxide cores can support notable TE wave propagation. Although both the $a_{b}$ and $s_{b}$ modes exhibit cutoff, TE waves can propagate several microns; distances can even exceed decay lengths observed for TM-polarized waves. A 100-nm-thick oxide core, for example, propagates $a_{b}$ TE-polarized light for $2 \mu \mathrm{m}$ at an excitation wavelength of $\lambda=400 \mathrm{~nm}$. In contrast, the $a_{b} \mathrm{TM}$ mode decays in about a quarter of the distance, propagating approximately $680 \mathrm{~nm}$. For both the $s_{b}$ and $a_{b}$ modes, bands of allowed propagation shift toward shorter wavelengths as oxide thickness is reduced. As wavelengths approach the regime of anomalous $\mathrm{Ag}$ dispersion, all modes become evanescent. Thin-film propagation, plotted in panels 6(b) and 6(d), does not exceed the nanometer scale for all wavelengths. However, like the $s_{b}$ TM-polarized modes of Fig. 5(b), a local maximum in propagation is observed for shorter wavelengths. This maximum increases with film thickness until 

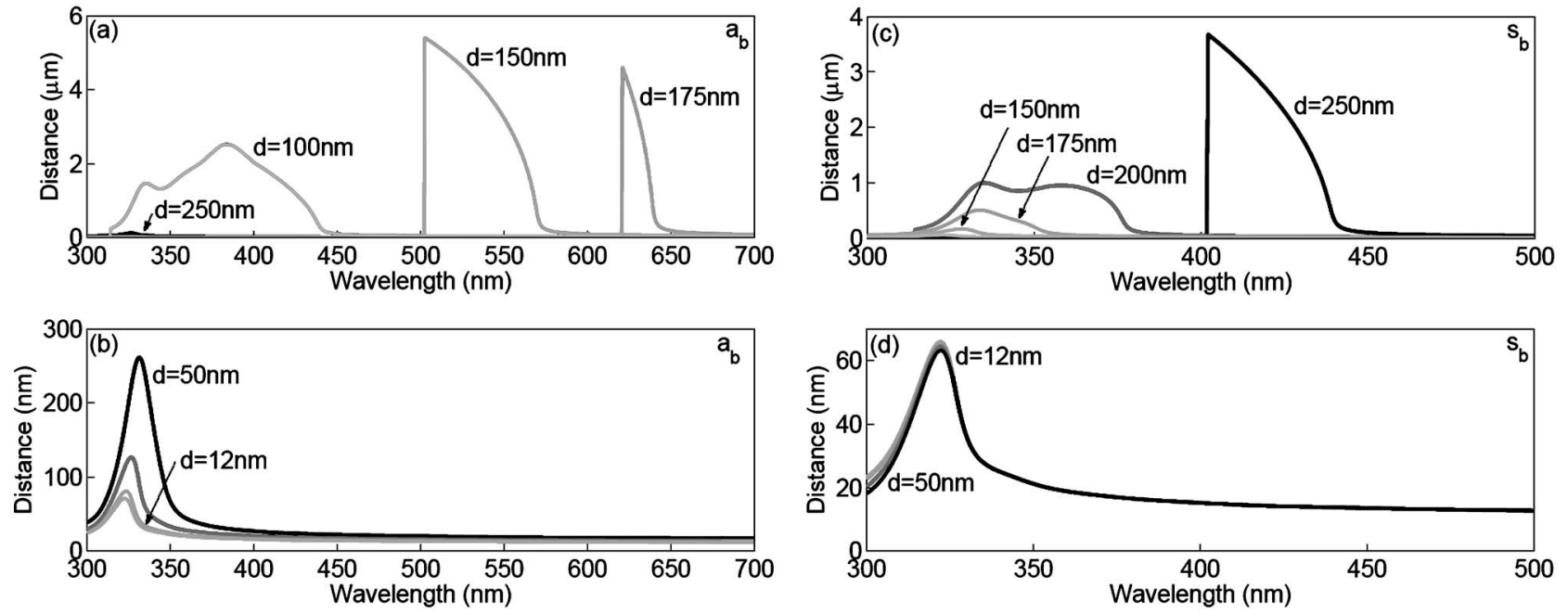

FIG. 6. MIM ( $\left.\mathrm{Ag} / \mathrm{SiO}_{2} / \mathrm{Ag}\right)$ TE-polarized propagation plotted as a function of wavelength for core thicknesses of $d=12 \mathrm{~nm}, 20 \mathrm{~nm}$, $35 \mathrm{~nm}, 50 \mathrm{~nm}, 100 \mathrm{~nm}, 150 \mathrm{~nm}, 175 \mathrm{~nm}, 200 \mathrm{~nm}$, and $250 \mathrm{~nm}$. Panels (a) and (b) plot propagation of the field antisymmetric modes; panels (c) and (d) plot propagation of the field symmetric modes. In panels (a) and (c), thicker oxide cores are seen to support propagating TE $a_{b}$ and $s_{b}$ waves. Although the modes exhibit cutoff, the propagating solutions can generate decay lengths comparable with TM solutions. In (b) and (d), the TE modes of thinner films $(d \leqslant 50 \mathrm{~nm})$ remain evanescent for all wavelengths. However, as $d$ approaches $100 \mathrm{~nm}$, conventional waveguiding modes can be accessed, and a region of enhanced propagation is observed for wavelengths of anomalous Ag dispersion.

the onset of conventional waveguiding is observed.

The combined results of Figs. 5 and 6 suggest that MIM modes can propagate many microns throughout a wide spectral range as core thicknesses are reduced from wavelength to subwavelength scales. For thicker films, this propagation results from a hybrid-polarized wave. As oxide thickness is decreased, the TE polarization is filtered, and only TM waves can propagate in the guide. This mixed TM-TE mode characteristic may be an important consideration in design of MIM tapered waveguides.

\section{MODE ENERGY DENSITY}

In an electrostatics limit, MIM waveguides locally serve as parallel-plate capacitors. At each metal-dielectric interface, localized charges will either interact with nearest neighbors in the conductor or across the dielectric. While the former situation is indicative of a surface charge wave, the latter suggests strong field concentration within the dielectric. Thus, unlike skin depth, the distribution of energy density in each media is a prime discriminator of wave propagation in MIM structures.

Figures 7 and 8 plot the electromagnetic energy density profiles of $\mathrm{Ag} / \mathrm{SiO}_{2} / \mathrm{Ag}$ structures as a function of distance from the waveguide median. Intensities have been normalized such that the power incident on the guide is equivalent for each mode. Within the dielectric core, energy density is defined in the usual way, with

$$
u_{\mathrm{SiO}_{2}}=\frac{1}{2}\left(\vec{E} \cdot \vec{D}^{*}+\vec{B} \cdot \vec{H}^{*}\right)=\frac{1}{2}\left(\varepsilon_{1} \vec{E} \cdot \vec{E}^{*}+\vec{B} \cdot \vec{B}^{*}\right),
$$

while outside the waveguide, the energy density is derived from Poynting's theorem in linear, lossy dispersive media ${ }^{28}$

$$
u_{\mathrm{Ag}}=\frac{1}{2}\left(\operatorname{Re}\left[\frac{d\left(\omega \varepsilon_{2}\right)}{d \omega}\right] \vec{E} \cdot \vec{E}^{*}+\vec{B} \cdot \vec{B}^{*}\right) .
$$

Figures 7(a) and 7(b) consider energy density both in the core and cladding for a 250-nm-thick oxide layer. Panel 7(a)
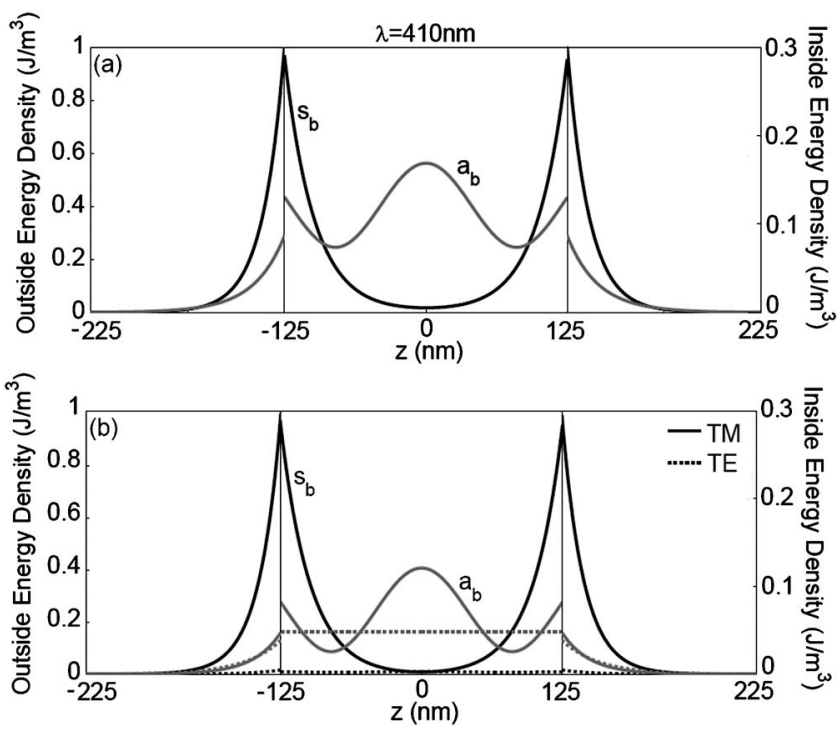

FIG. 7. Normalized electromagnetic energy density profiles plotted as a function of distance from the waveguide median $(z$ $=0)$ for $\mathrm{MIM}\left(\mathrm{Ag} / \mathrm{SiO}_{2} / \mathrm{Ag}\right)$ structures with core thickness $d$ $=250 \mathrm{~nm}$. Note that energy density axes relevant for the metal are shown on the left while those relevant for the oxide are shown on the right. (a) Total energy density (TE+TM polarizations) for the antisymmetric ( $a_{b}$, gray) and symmetric $\left(s_{b}\right.$, black) dispersion solutions. (b) Separate TE (dotted) and TM (solid) energy density contributions for the $a_{b}$ (gray) and $s_{b}$ (black) modes. In each panel, the excitation wavelength has been set to $\lambda=410 \mathrm{~nm}$. 

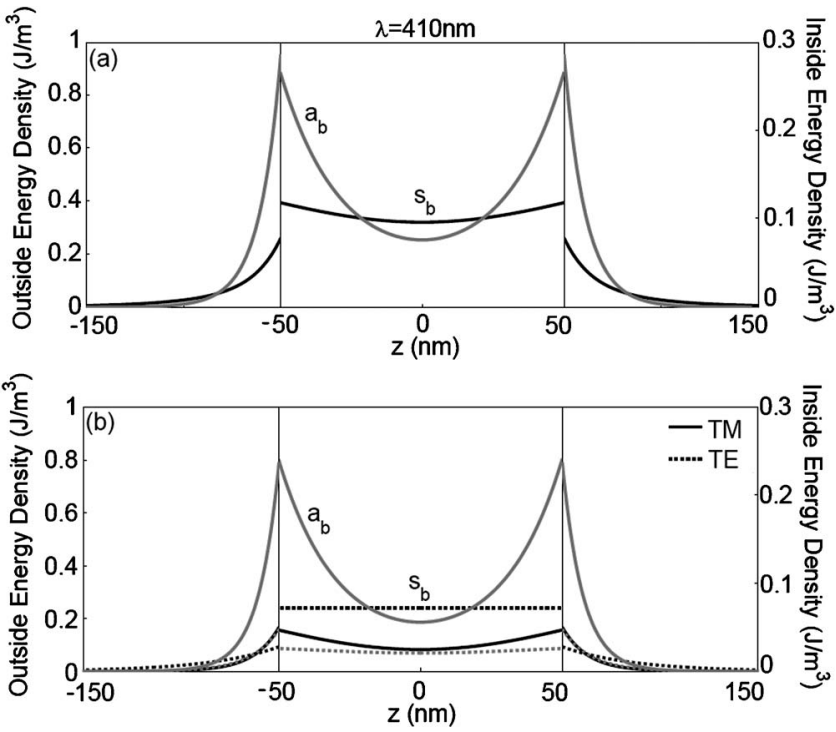

FIG. 8. Normalized electromagnetic energy density profiles plotted as a function of distance from the waveguide median $(z$ $=0)$ for $\mathrm{MIM}\left(\mathrm{Ag} / \mathrm{SiO}_{2} / \mathrm{Ag}\right)$ structures with core thickness $d$ $=100 \mathrm{~nm}$. Again, energy density axes relevant for the metal are shown on the left while those relevant for the oxide are shown on the right. (a) Total energy density (TE+TM polarizations) for the antisymmetric ( $a_{b}$, gray) and symmetric $\left(s_{b}\right.$, black) dispersion solutions. (b) Separate TE (dotted) and TM (solid) energy density contributions for the $a_{b}$ (gray) and $s_{b}$ (black) modes.

plots the total energy density (TE+TM contributions) at a free-space wavelength of $410 \mathrm{~nm}(\sim 3.2 \mathrm{eV})$. Panel 7(b) illustrates the separate contributions of each polarization. Note that energy density values in the cladding are indicated on the left scale of the plot; values inside the core are shown on the right.

As seen in Fig. 7(a), total energy density of the $a_{b}$ wave is reminiscent of conventional waveguiding modes, with high density concentrations both in the waveguide and at the metal surface. In the waveguide center, the density profile is nearly parabolic, reaching a maximum value at $z=0$. Outside the guide, energy density decays exponentially from the interface with a decay length of $16 \mathrm{~nm}$. In contrast, total energy density of the $s_{b}$ wave is reminiscent of a surface mode, with high electromagnetic densities concentrated at the interface. Inside the guide, intensity exhibits a minimum at $z=0$ and attains maximal amplitude at the $\mathrm{Ag} / \mathrm{SiO}_{2}$ interface. Outside the core, energy densities decay within $11 \mathrm{~nm}$ of the interface. Such results suggest that the long-ranging modes of MIM waveguides generally have high densities at the metal-dielectric interface, in analogy with surface electromagnetic waves.

Figure 7(b) plots the separate TM and TE contributions to energy density for a $250 \mathrm{~nm} \mathrm{SiO}_{2}$ core, holding wavelength fixed at $\lambda=410 \mathrm{~nm}$. TM contributions are plotted using solid-line type; TE contributions are dotted. Additionally, $s_{b}$ modes are shown in black and $a_{b}$ modes are plotted in gray. As the figure illustrates, TM densities mimic those observed in panel 7(a). Intensity of the TE $a_{b}$ mode is constant throughout the waveguide core, while intensity of the TE $s_{b}$ mode is minimal throughout the waveguide and functionally behaves much like the TM $a_{b}$ mode. Interestingly, the TE $a_{b}$ wave represents the only polarization that does not propagate several microns in the waveguide. This observation is supported by decay lengths of energy density in the Ag: the TE $a_{b}$ mode extends to $20 \mathrm{~nm}$ in the $\mathrm{Ag}$, while decay lengths for the TE $s_{b}$ and TM $a_{b}$ and $s_{b}$ modes are, respectively, $14 \mathrm{~nm}$, $12.8 \mathrm{~nm}$, and $14 \mathrm{~nm}$.

Figures 8(a) and 8(b) plot energy densities in the core and cladding for a 100 -nm-thick $\mathrm{SiO}_{2}$ oxide layer. Again, wavelength is held fixed at $410 \mathrm{~nm}$, and both total and contributed energy density profiles are considered. As seen in Fig. 8(a), both $a_{b}$ and $s_{b}$ modes are concave-up, exhibit a minimum at $z=0$, and reach a maximum at the $\mathrm{Ag} / \mathrm{SiO}_{2}$ interface. The $a_{b}$ mode decays within $11 \mathrm{~nm}$ outside the waveguide core (contrasted with a $20 \mathrm{~nm}$ decay length for the $s_{b}$ mode), explaining the enhanced propagation lengths observed for this mode. [Recall that at $\lambda=410 \mathrm{~nm}$, the $a_{b}$ mode propagates $\sim 1 \mu \mathrm{m}$ (TM) or $1.7 \mu \mathrm{m}$ (TE) while the $s_{b}$ mode propagates $\sim 0.5 \mu \mathrm{m}(\mathrm{TM})$ or $20 \mathrm{~nm}$ (TE).] Figure 8(b) reveals that TE and TM modes are of competing magnitudes inside the waveguide. The equivalence in form and magnitude of the TE and TM $a_{b}$ modes reflects their comparable propagation lengths at this wavelength. The TE $s_{b}$ polarization is the only nonpropagating field at $\lambda=410 \mathrm{~nm}$, and exhibits constant intensity within the core. As with the $250 \mathrm{~nm}$ oxide guide, near-constant core energy density is accompanied by large energy density decay lengths in the metal. The TE $s_{b}$ polarization extends $42 \mathrm{~nm}$ into the $\mathrm{Ag}$, while density decay lengths for the TE $a_{b}$ and TM $a_{b}$ and $s_{b}$ modes are, respectively, $14.5 \mathrm{~nm}, 10.5 \mathrm{~nm}$, and $13 \mathrm{~nm}$.

Interestingly, the nonpropagating $\mathrm{TE} s_{b}$ polarization achieves higher energy density values at the waveguide median than any other polarization. Here, energy densities in the oxide approach $80 \%$ of those observed in the metal (for the same mode). As film thickness is decreased through $12 \mathrm{~nm}, \mathrm{TE}$ intensities in the core can equal and even exceed maximal Ag energy densities. Such results suggest that the propagating modes of subwavelength structures are generally surface modes, with field localization dominant within a narrow band about the metal-dielectric interface. However, the core energy densities of nonpropagating TE modes can rival the intensities observed in resonantly excited metal nanoparticle arrays. ${ }^{29}$ This interdependence of energy density, mode polarization, and oxide thickness suggests potential for both waveguiding and field-sensitive applications, including biological sensing.

\section{CONCLUSIONS}

Device architectures of present are reliant on indexcontrasted media for signal storage and transmission. Accordingly, conventional waveguides are both well understood and heavily utilized for light propagation on macroscopic scales. However, as device sizes are scaled to theoretical limits, light-matter interactions must be tuned to support modes within nanoscopic dimensions. Conversion of the photon mode into a surface plasmon mode is one such mechanism for subwavelength-scale signal transmission.

In thin metallic films, surface plasmons can propagate over tens of centimeters at infrared wavelengths. However, 
this long-range propagation is achieved at the expense of confinement: field penetration increases exponentially from the metal-dielectric interface, extending over several microns into the surrounding dielectric. In contrast, the skin depth of MIM structures is limited by optical decay lengths in the metal. As the waveguide core is reduced to nanometer sizes, the structure still supports propagation over $10 \mu \mathrm{m}$, with fields confined to within $20 \mathrm{~nm}$ of the structure.

Depending on transverse dimensions, MIM waveguides can support both conventional and plasmonic modes; cutoff wave vectors are not observed until the core diameter is reduced below $\sim 20 \mathrm{~nm}$ or exceeds $\sim 100 \mathrm{~nm}$. This superposition of modes results in wide tunability of energy density throughout the electromagnetic spectrum. While energy densities are generally high at the metal interface, intensities within the waveguide can be comparable to values observed in nanoparticle array gaps.

Judicious arrangement of IMI and MIM plasmon waveguides promises potential for two-dimensional planar loss-localization balance. When combined with the recent remarkable progress in nanoscale fabrication, the aforementioned results might inspire an alternative class of waveguide architectures-ultimately, a class of subwavelength plasmonic interconnects, not altogether different from the silicon-on-insulator networks of today.

\section{ACKNOWLEDGMENTS}

The authors would like to thank Henri Lezec and Domenico Pacifici for thoughtful and engaging discussions related to this work. Financial support at Caltech was provided by the Air Force Office of Scientific Research, MURI Grant No. FA9550-04-1-0434. One of us (JAD) acknowledges fellowship support from the National Science Foundation and the Department of Defense Army Research Office. Work at AMOLF is part of the research program of FOM and is financially supported by NWO.
*Electronic address: jdionne@caltech.edu

${ }^{1}$ S. A. Maier, P. G. Kik, H. A. Atwater, S. Meltzer, E. Harel, B. E. Koel, and A. A. G. Requicha, Nat. Mater. 2, 229 (2003).

${ }^{2}$ D. Sarid, Phys. Rev. Lett. 47, 1927 (1981).

${ }^{3}$ J. J. Burke, G. I. Stegeman, and T. Tamir, Phys. Rev. B 33, 5186 (1986).

${ }^{4}$ J. A. Dionne, L. A. Sweatlock, A. Polman, and H. A. Atwater, Phys. Rev. B 72, 075405 (2005) and references therein.

${ }^{5}$ T. Nikolajsen, K. Leosson, I. Salakhutdinv, and S. I. Bozhevolnyi, Appl. Phys. Lett. 82, 668 (2003).

${ }^{6}$ S. Schultz, D. Smith, J. Mock, and D. A. Schultz, Proc. Natl. Acad. Sci. U.S.A. 97, 996 (2000).

${ }^{7}$ L. R. Hirsch, J. B. Jackson, A. Lee, N. J. Halas, and J. L. West, Anal. Chem. 75, 2377 (2003).

${ }^{8}$ A. McFarland and R. Van Duyna, Nano Lett. 3, 1057 (2003).

${ }^{9}$ A. Haes and R. Van Duyne, Proc. SPIE 5221, 47 (2003).

${ }^{10}$ A. Haes and R. Van Duyne, Anal. Bioanal. Chem. 379, 920 (2004)

${ }^{11}$ J. Gersten and A. Nitzan, J. Chem. Phys. 73, 3023 (1980).

${ }^{12}$ F. J. Garcia-Vidal and J. B. Pendry, Phys. Rev. Lett. 77, 1163 (1996).

${ }^{13}$ S. L. Zou and G. C. Schatz, Chem. Phys. Lett. 403, 62 (2005).

${ }^{14}$ G. I. Stegeman, J. J. Burque, and D. G. Hall, Appl. Phys. Lett. 41, 906 (1982).

${ }^{15}$ S. I. Bozhevolnyi and V. Z. Lozovski, Phys. Rev. B 65, 235420 (2002).

${ }^{16}$ Y. Shen and P. N. Prasad, Appl. Phys. B 74, 641 (2002).

${ }^{17}$ H. Tuovinen and M. Kauranen, J. Nonlinear Opt. Phys. Mater. 11, 421 (2002).

${ }^{18}$ S. Baher and M. G. Cottam, Surf. Rev. Lett. 10, 13 (2003).

${ }^{19}$ For a comprehensive review of surface plasmon properties, see $\mathrm{H}$.
Raether, Surface Plasmons on Smooth and Rough Surfaces and on Gratings (Springer-Verlag, Berlin, 1988).

${ }^{20}$ V. Almeida, Q. Xu, C. Barrios, and M. Lipson, Opt. Lett. 29, 1209 (2004).

${ }^{21}$ E. N. Economou, Phys. Rev. 182, 539 (1969).

${ }^{22}$ R. Zia, M. D. Selker, P. B. Catrysse, and M. L. Brongersma, J. Opt. Soc. Am. A 21, 2442 (2004).

${ }^{23}$ P. B. Johnson and R. W. Christy, Phys. Rev. B 6, 4370 (1972).

${ }^{24}$ Handbook of Optical Constants of Solids, edited by E. Palik (Academic Press, Inc., New York, 1985).

${ }^{25}$ In this context, use of the term "bound" is meant to be in analogy with the bound modes of IMI plasmon waveguides. However, since MIM modes below the SP resonance do not necessarily lie below the light line, a more appropriate descriptor might be "hybrid" or "mixed" modes.

${ }^{26}$ While skin depth calculations for forbidden modes are of no physically relevant consequence, they do indicate field penetration depths of the diffracted wave. In particular, even though $k_{x}=0$ for forbidden modes, $k_{z}$ can still assume a finite value.

${ }^{27}$ This observation is further supported by the data for thin waveguides $(d<100 \mathrm{~nm})$, presented in Fig. 5. For the antisymmetric mode [Fig. 5(a)], propagation increases with $d$ while skin depth remains constant. In contrast, for the symmetric mode [Fig. 5(b)], propagation and skin depth both increase with $d$. As indicated in the section's conclusion, these results point towards interference-not absorption-as the prime loss mechanism.

${ }^{28}$ L. D. Landau and E. M. Lifshitz, Electrodynamics of Continuous Media, 2nd ed. (Butterworth-Heinenann, Oxford, 1984).

${ }^{29}$ L. A. Sweatlock, S. A. Maier, H. A. Atwater, J. J. Penninkhof, and A. Polman, Phys. Rev. B 71, 235408 (2005). 